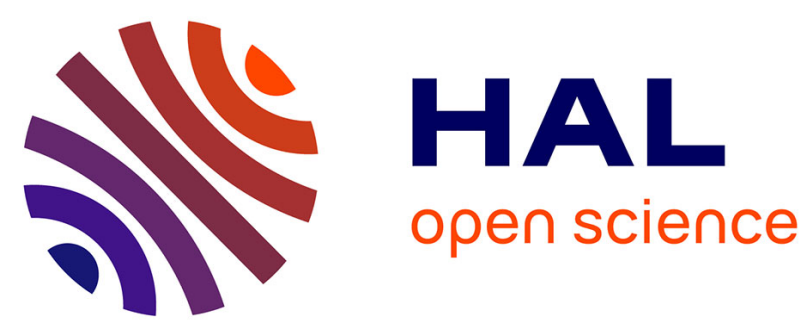

\title{
On the Number of Attractors of Positive and Negative Boolean Automata Circuits.
}

\author{
Jacques Demongeot, Mathilde Noual, Sylvain Sené
}

\section{To cite this version:}

Jacques Demongeot, Mathilde Noual, Sylvain Sené. On the Number of Attractors of Positive and Negative Boolean Automata Circuits.. 24th IEEE International Conference on Advanced Information Networking and Applications Workshops (WAINA 2010), Apr 2010, Perth, WA, Australia. pp.782789, 10.1109/WAINA.2010.141 . hal-00647877

\section{HAL Id: hal-00647877 https://hal.science/hal-00647877}

Submitted on 14 Feb 2014

HAL is a multi-disciplinary open access archive for the deposit and dissemination of scientific research documents, whether they are published or not. The documents may come from teaching and research institutions in France or abroad, or from public or private research centers.
L'archive ouverte pluridisciplinaire HAL, est destinée au dépôt et à la diffusion de documents scientifiques de niveau recherche, publiés ou non, émanant des établissements d'enseignement et de recherche français ou étrangers, des laboratoires publics ou privés. 


\title{
On the number of attractors of positive and negative Boolean automata circuits
}

\author{
Jacques Demongeot ${ }^{1}$, Mathilde Noual ${ }^{2,4}$, and Sylvain Sené 3,4 \\ 1 Université Grenoble 1, TIMC-IMAG, CNRS UMR5525, 38706 La Tronche, France \\ 2 Université de Lyon, ÉNS-LYON, LIP, CNRS UMR5668, 69007 Lyon, France \\ 3 Université d'Évry - Val d'Essonne, IBISC, CNRS FRE3190, 91000 Evry, France \\ 4 Institut rhône-alpin des systèmes complexes, IXXI, 69007 Lyon, France
}

\begin{abstract}
In line with fields of theoretical computer science and biology that study Boolean automata networks often seen as models of regulation networks, we present some results concerning the dynamics of networks whose underlying interaction graphs are circuits, that is, Boolean automata circuits. In the context of biological regulation, former studies have highlighted the importance of circuits on the asymptotic dynamical behaviour of the biological networks that contain them. Our work focuses on the number of attractors of Boolean automata circuits. We prove how to obtain formally the exact value of the total number of attractors of a circuit of arbitrary size $n$ as well as, for every positive integer $p$, the number of its attractors of period $p$ depending on whether the circuit has an even or an odd number of inhibitions. As a consequence, we obtain that both numbers depend only on the parity of the number of inhibitions and not on their distribution along the circuit.

Keywords: Discrete dynamical system, formal neural network, positive and negative circuit, asymptotic behaviour, attractor.
\end{abstract}

\section{Introduction}

The theme of this article is set in the general framework of complex dynamical systems and, more precisely, that of regulation or neural networks modeled by means of discrete mathematical tools. Since McCulloch and Pitts [1] proposed threshold Boolean automata networks to represent neural networks formally in 1943 and later, at the end of the 1960's, Kauffman [2] and Thomas [3] introduced the first models of genetic regulation networks, many other studies based on the same or different formalisms were carried out on the theoretical properties of such networks. One of the main motivations of many of them was to better understand those emergent dynamical behaviours that networks display and that cannot be explained or predicted by a simple analysis of the local interactions existing between the components of the networks. Amongst the studies published in this context since the end of the 1990's, one may cite $[4,5,6,7,8]$. Earlier on, Hopfield $[9,10]$ emphasised the notions of memory and learning and Goles et al. revealed in $[11,12,13]$ interesting dynamical properties of some particular networks. Further, later works by Thomas and Kauffman $[14,15]$ yielded conjectures and gave rise to problematics that are still relevant in the field of regulation 
networks beyond the particular definition of the models one may choose to use. For instance, Thomas highlighted the importance of specific patterns on the dynamics of discrete regulation networks and Kauffman gave an approximation of the number of different possible asymptotic behaviours of Boolean networks.

From the point of view of theoretical biology as well as that of theoretical computer science, it seems to be of great interest to address the question of the number of attractors in the dynamics of a network. Close to the 16th Hilbert problem concerning the number of limit cycles of dynamical systems [16], this question has already been considered in some works $[17,18,19]$. It is of particular relevance in the neighbouring field of formal neural networks where attractors represent memorisation capacity $[9,10]$. Driven by a similar will to understand the dynamical properties of (regulation or neural) networks modeled by Boolean automata networks, we have decided to first focus our attention on a simple instance of Boolean automata networks, that is, Boolean automata circuits (which also happen to be a simple instance of threshold Boolean automata networks [1]). The reason for this choice is that circuits are known to play an important part in the dynamics of a network that contains them. One way to see this is to note that a network whose underlying interaction graph is a tree or more generally a graph without circuits can only eventually end up in a configuration that will never change over time. A network with retroactive loops, on the contrary, will exhibit more diverse dynamical behaviour patterns. Thomas [14] had already noted the importance of underlying circuits in networks. He formulated conjectures concerning the role of positive (i.e., with an even number of inhibitions) and negative (i.e., with an odd number of inhibitions) circuits in the dynamics of regulation networks. Besides the fact that they are known to be decisive patterns for the dynamics of arbitrary biological networks, circuits are also relevant because they may be regarded specifically as internal layers of feedforward networks ${ }^{5}$. Identifying the dynamics of circuits is thus a first step in the process of understanding and formalising the dynamics of such networks. The reason why feedforward networks present a theoretical interest is that many biological systems are known to have a feedforward architecture [20]. For instance, in the lamprey $[21,22]$, the sensory input and the basal ganglia control network respectively activates and inhibits the locomotor network without any feedback of the latter to them. It is also the case in the hypothalamus in the feed-forward chains of Glu- and Gaba-neurons [23].

In this paper, we give the results and the proofs that allow a new characterisation of the asymptotic dynamical behaviour of Boolean automata circuits evolving synchronously (i.e. at each time step, every node executes its transition function), in terms of combinatorics. After the preliminary section 2, sections 3 and 4 deal, respectively, with the dynamics of positive circuits and negative circuits updated synchronously. For both types of circuits, we obtain the exact values of the total number of attractors of these circuits and of their number of attractors of period $p$ for every positive integer $p$. These values happen to

\footnotetext{
${ }^{5}$ Feedforward networks are networks whose structure can be represented by a layered graph with no feedback loops between layers.
} 
be terms of integer sequences defined by different combinatoric problems that we showed to be isomorphic to the problem of counting attractors of a circuit. Section 5 mentions some of them. The conclusion discusses the main perspective of this work.

\section{Definitions, notations and preliminary results}

A circuit of size $n$ is a directed graph that we will denote by $\mathbb{C}_{n}=(V, A)$. We will consider that its set of nodes, $V=\{0, \ldots, n-1\}$, corresponds to the the set of elements of $\mathbb{Z} / n \mathbb{Z}$ so that, considering two nodes $i$ and $j, i+j$ designates the node $i+j \bmod n$. The circuits set of arcs is then $A=\{(i, i+1) \mid 0 \leq i<n\}$. Let $i d$ be the identity function $(\forall a \in\{0,1\}, i d(a)=a)$ and neg the negation function $(\forall a \in\{0,1\}$, neg $(a)=\neg a=1-a)$. A Boolean automata network of size $n$ associated to a circuit or Boolean automata circuit of size $n$ is a couple $R_{n}=\left(\mathbb{C}_{n}, F\right)$ where $\mathbb{C}_{n}$ is a circuit of size $n$ whose nodes are assimilated to the automata of the network and $F$ is the global transition function of the network. By a minor abuse of language, we will refer to the (global) state of $R_{n}$ as a vector $x=\left(x_{0} \ldots x_{n-1}\right) \in\{0,1\}^{n}$ whose coefficient $x_{i}$ is the state of node $i$ of $\mathbb{C}_{n} . F$ is defined by a set of $n$ local transition functions $\left\{f_{i} \in\{i d, n e g\} \mid 0 \leq i<n\right\}$ that will be, for our matter, applied synchronously: let $x=\left(x_{0} \ldots x_{n-1}\right) \in\{0,1\}^{n}$ represent a global state of $R_{n}$, then:

$$
F(x)=\left(f_{0}\left(x_{n-1}\right), \ldots, f_{i}\left(x_{i-1}\right), \ldots, f_{n-1}\left(x_{n-2}\right)\right) .
$$

When there will be no ambiguity as to what network we are considering, we will also note this transition rule $x(t+1)=F(x(t))$ where $x(t) \in\{0,1\}^{n}$ and $t \in \mathbb{N}$ so that $\forall k \in \mathbb{N}, x(t+k)=F\left(F^{k-1}(x(t))\right)$ and at the local level of nodes, $x_{i}(t+1)=F(x(t))_{i}=f_{i}\left(x_{i-1}(t)\right)$. Note that with the restriction on the local transition functions, $f_{i} \in\{i d, n e g\}$, we do not loose any generality. Indeed, if at least one of the nodes of the circuit, say node $i$, has a constant local function then its incoming arc is useless. Node $i$ does not depend on node $i-1$ and we no longer are looking at a "real" circuit. An arc $(i, i+1)$ is said to be positive (resp. negative) if $f_{i+1}=i d$ (resp. $f_{i+1}=n e g$ ). The network $R_{n}$ and the circuit associated, $\mathbb{C}_{n}=(V, A)$, are said to be positive (resp. negative) if the number of negative arcs of $A$ is even (resp. odd).

Note that the definition that we have just given of Boolean automata network associated to a circuit, or, more simply Boolean automata circuit, is a particular example of a quasi-minimal ${ }^{6}$ threshold Boolean automata network. Indeed, as it can easily be checked, $i d$ and neg can both be expressed as threshold functions and thus, so can all local transition functions using identical interaction weights and threshold values for all arcs and nodes (see [5] for more details on this subject).

\footnotetext{
${ }^{6}$ A quasi-minimal network is a network such that, if an arc is removed from the associated graph, then the dynamics of the network is changed.
} 
Let $R_{n}=\left(\mathbb{C}_{n}, F\right)$ be a Boolean automata circuit of size $n$. In the sequel, we will make substantial use of the following function:

$$
F[j, i]= \begin{cases}f_{j} \circ f_{j-1} \circ \ldots \circ f_{i} & \text { if } i \leq j \\ f_{j} \circ f_{j-1} \circ \ldots \circ f_{0} \circ f_{n-1} \circ \ldots \circ f_{i} & \text { if } j<i\end{cases}
$$

There are several things to note about this function. First, because $\forall k, f_{k} \in$ $\{i d, n e g\}, F[j, i]$ is injective. Second, if $\mathbb{C}_{n}$ is positive then $\forall j, F[j+1, j]=i d$ and if, on the contrary, $\mathbb{C}_{n}$ is negative then $\forall j, F[j+1, j]=n e g$. Finally, it is also important to notice that the following is true for all $t \in \mathbb{N}, p \leq n, i \in \mathbb{Z} / n \mathbb{Z}$ :

$$
\begin{aligned}
x_{i}(t+p)= & \\
& f_{i}\left(x_{i-1}(t+p-1)\right)=f_{i}\left(f_{i-1}\left(x_{i-2}(t+p-2)\right)\right)= \\
& \quad \ldots=F[i, i-p+1]\left(x_{i-p}(t)\right) .
\end{aligned}
$$

Since the set of global states of $R_{n}$ is finite, when the network is updated, it necessarily ends up looping. In other words, $\forall x(0) \in\{0,1\}^{n}, \exists t, p, x(t+$ $p)=x(t)$. We call attractor or limit cycle the orbit of $x(t)$, i.e., the finite set $\{x(t+k) \mid k \in \mathbb{N}\}$. The period of this attractor is its cardinal, i.e., the smallest $p$ such that for any state $k \in \mathbb{N}, x(t+k+p)=x(t+k)$. Elements belonging to an attractor of period 1 are usually called fixed points. The set of all global states of $R_{n}$ belonging to an attractor of period $p$ is denoted by:

$$
\mathcal{S}_{p}\left(R_{n}\right)=7\left\{x \in\{0,1\}^{n} \mid F^{p}(x)=x \text { and } \forall d<p, F^{d}(x) \neq x\right\}
$$

and the number of attractors of period $p$ of a network $R_{n}$ is denoted by:

$$
\mathrm{A}_{p}\left(R_{n}\right)=\frac{1}{p} \cdot\left|\mathcal{S}_{p}\left(R_{n}\right)\right|
$$

Figure 1 pictures three different circuits of size 4 (two positive and one negative) as well as their dynamics by an iteration graph whose set of vertices is $\{0,1\}^{4}$ and whose strongly connected components are the attractors of the network.

Now, let $R_{n}=\left(\mathbb{C}_{n}, F\right)$. We define the following property $\mathcal{P}_{F}$ on $\{1, \ldots, n\} \times$ $\{0,1\}^{n}$ depending on $F$ :

$$
\forall p \leq n, \forall x \in\{0,1\}^{n}, \quad
$$

By induction on $k$ where $i=k \cdot p+r, r<p$, it can be shown that property $\mathcal{P}_{F}$ is equivalent to the following:

$$
\mathcal{P}_{F}(p, x) \Leftrightarrow\left\{\begin{array}{l}
\forall i<p, x_{i}=F[i, i-p+1]\left(x_{i-p}\right) \text { and } \\
\forall i \geq p, x_{i}=F[i,(i \bmod p)+1]\left(x_{i \bmod p}\right)
\end{array}\right.
$$




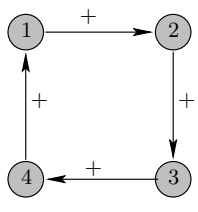

1.a.

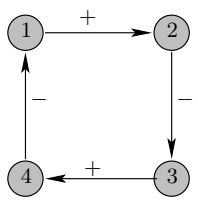

1.b.

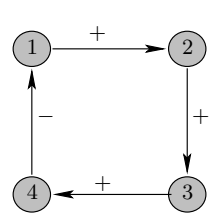

1.c.

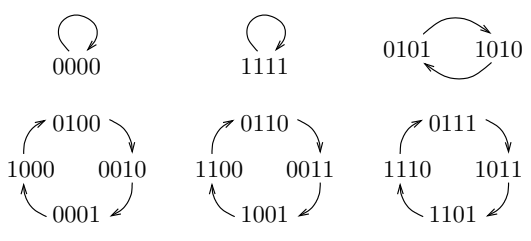

2.a.

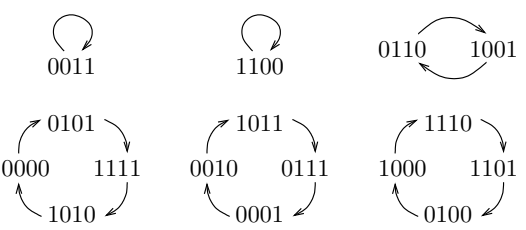

2.b.

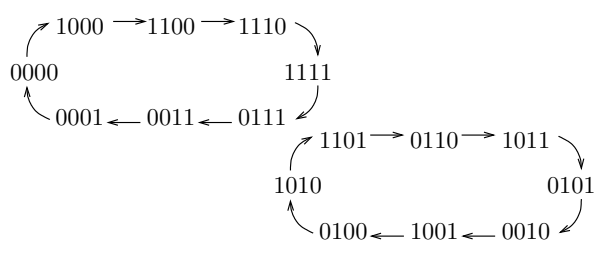

2.c.

Fig. 1. Figures 1.a., b. and $c$. represent three different Boolean automata circuits of size $n=4$. That of figures 1.a. and b. are positive while that of figure 1.c. is negative. Figures 2.a.,b. and $c$. picture the iteration graph of these networks, that is, the graph that represents their dynamics. The nodes of these graphs are the elements of $\{0,1\}^{n}$ and an arc (x,y) exists in this graph if and only if $F(x)=y$. Every strongly connected component of the iteration graph corresponds to an attractor of size the number of elements in this component. Note that in all three cases here, all elements belong to an attractor. This is usually not the case with arbitrary Boolean automata networks which are not circuits.

The definition of $\mathcal{P}_{F}(p, x)$ takes its meaning from the following result which characterises global states that loop after $p$ transitions (or less), i.e. states of $\mathcal{S}_{d}$ where $d \leq p$ :

Lemma 1. Let $R_{n}=\left(\mathbb{C}_{n}, F\right)$ be a Boolean automata circuit of size $n$, let $p \leq n$ and let $x(0) \in\{0,1\}^{n}$ be an arbitrary global state of $R_{n}$. Then,

$$
\forall t, x(t)=x(t+p) \Leftrightarrow \forall t, \mathcal{P}_{F}(p, x(t)) .
$$

Proof. Suppose that $\forall t, \mathcal{P}_{F}(p, x(t))$. Then,

$$
\begin{aligned}
& \forall r, 0 \leq r<p, F[p+r, r+1]\left(x_{r}(t)\right)= \\
& x_{p+r}(t+p)=F[p+r, r+1]\left(x_{r}(t+p)\right),
\end{aligned}
$$


where the first equality is always true (see the remark made above after the definition of $F[j, i])$ and the second is due to $\mathcal{P}_{F}(p, x(t+p))$. With the injectivity of $F[p+r, r+1]$, this implies that $x_{r}(t)=x_{r}(t+p)$. In addition, $\forall i, p \leq i<n$ such that $r=i \bmod p$ :

$$
x_{i}(t+p)=F[i, r+1]\left(x_{r}(t+p)\right)=F[i, r+1]\left(x_{r}(t)\right)=x_{i}(t) .
$$

The first equality above is due to $\mathcal{P}_{F}(p, x(t+p))$, the second to $x_{r}(t+p)=x_{r}(t)$ and the third to $\mathcal{P}_{F}(p, x(t))$. On the other hand, suppose that $\forall t, x(t)=x(t+p)$. Then, $\forall i \in \mathbb{Z} / n \mathbb{Z}, x_{i}(t)=x_{i}(t+p)=F[i, i-p+1]\left(x_{i-p}(t)\right)$.

In the sequel, we will compare the dynamics of particular couples of circuits of same signs, $R_{p}=\left(\mathbb{C}_{p}, H\right)$ and $R_{n}=\left(\mathbb{C}_{n}, F\right)$, where $p$ divides $n=p \cdot q$ and where the global transition function $H$ of $R_{p}$ is defined by the set of local transition functions $\left\{h_{i} \mid i \in \mathbb{Z} / p \mathbb{Z}\right\}$ and the global transition function $F$ of $R_{n}$ is defined by the set $\left\{f_{i} \mid i \in \mathbb{Z} / n \mathbb{Z}\right\}$. More precisely, we will build an isomorphism between the iteration graphs of $R_{n}$ and $R_{p}$. To do this, we will use the injection $\mathcal{Q}_{F, H}$ defined below that maps a state $x(t) \in\{0,1\}^{p}$ of $R_{p}$ to a state $y(t)=\mathcal{Q}_{F, H}(x(t)) \in\{0,1\}^{n}$ of $R_{n}$ such that $\forall k \in \mathbb{N}, x_{0}(t+k)=y_{0}(t+k)$. The idea behind the definition of $\mathcal{Q}_{F, H}$ is roughly to make $R_{n}$ "mimic" the dynamical behaviour of $R_{p}$.

$\forall x \in\{0,1\}^{p}, \forall i=k p+r \in \mathbb{Z} / n \mathbb{Z}$ such that $r=i \bmod p$ and $s=i+1 \bmod p$,

$$
\mathcal{Q}_{F, H}(x)_{i}=\left\{\begin{aligned}
x_{r} & \text { if } f_{i+1}=h_{s} \text { and } y_{i+1}=x_{s} \\
& \text { or } f_{i+1} \neq h_{s} \text { and } y_{i+1} \neq x_{s} \\
& \text { or } i=r=0 \\
\neg x_{r} & \text { if } f_{i+1}=h_{s} \text { and } y_{i+1} \neq x_{s} \\
& \text { or } f_{i+1} \neq h_{s} \text { and } y_{i+1}=x_{s}
\end{aligned}\right.
$$

By a reversed induction on $i$, the following can be shown:

$$
\begin{aligned}
\mathcal{Q}_{F, H}(x)_{0}=x_{0} \text { and } \forall 0<i<n, \\
\qquad \mathcal{Q}_{F, H}(x)_{i}= \begin{cases}x_{r} & \text { if } F[0, i+1]=H[0,1]^{q-1-k} \circ H[0, s] \\
\neg x_{r} & \text { otherwise }\end{cases}
\end{aligned}
$$

Note that $H[0,1]=i d$ if and only $R_{p}$ is positive. In the sequel, we will only consider one of the two following cases (the reason for this will be given further on):

1. $R_{p}$ and $R_{n}$ are both positive or

2. $R_{p}$ and $R_{n}$ are both negative and $q$ is odd.

In both cases, it holds that $F[0,1]=H[0,1]$ and $H[0,1]^{q-1}=i d$ so that $\mathcal{Q}_{F, H}(x)_{0}=x_{0}$. The reader can also check that $F\left(\mathcal{Q}_{F, H}(x)\right)_{i+1}=H\left(\mathcal{Q}_{F, H}(x)\right)_{s} \Leftrightarrow$ $\mathcal{Q}_{F, H}(x)_{i+1}=x_{s}$ so that $F\left(\mathcal{Q}_{F, H}(x)\right)=\mathcal{Q}_{F, H}(H(x))$. Since $\mathcal{Q}_{F, H}$ is clearly injective, in both cases mentioned, $\mathcal{Q}_{F, H}$ satisfies the desired properties. 


\section{Positive circuits}

In this section, we focus on positive Boolean automata circuits, that is, networks associated to circuits having an even number of negative arcs.

Lemma 2. Let $R_{n}=\left(\mathbb{C}_{n}, F\right)$ be a positive Boolean automata circuit of size $n$. Every global state $x \in\{0,1\}^{n}$ of $R_{n}$ belongs to an attractor of period a divisor of $n$.

Proof. Suppose that $F$ is defined by $\left\{f_{i} \mid i \in \mathbb{Z} / n \mathbb{Z}\right\}$. Let $x(t)=\left(x_{0}(t), \ldots, x_{n-1}(t)\right)$ be an arbitrary global state of $R_{n}$. Then,

$$
\forall j \in \mathbb{Z} / n \mathbb{Z}, x_{j}(t+n)=F[j, j+1]\left(x_{j}(t+n-n)\right)=x_{j}(t) .
$$

The last equality above holds because, $\mathbb{C}_{n}$ being positive, $F[j, j+1]=i d$.

Lemma 2 is an extension of a result proven by Goles et al. in [24]. The purpose of the two following lemmas is to compare the dynamics of different circuits of same sign. The first of these two results, Lemma 3, is proven by establishing an isomorphism between the attractors whose period is the largest of two networks of same size. Lemma 4 is proven by establishing an isomorphism between the attractors of circuits of different sizes. Very roughly, the main idea in the construction of these isomorphisms is to show that the dynamical behaviour of one of the circuits can always be made to "mimic" that of the other.

Lemma 3. If $R_{p}=\left(\mathbb{C}_{p}, F\right)$ and $R_{p}^{\prime}=\left(\mathbb{C}_{p}^{\prime}, H\right)$ are two positive Boolean automata circuits, both of size $p$, then the number of attractors of period $p$ of both networks is the same:

$$
A_{p}\left(R_{p}\right)=A_{p}\left(R_{p}^{\prime}\right)
$$

Proof. Let $x(t) \in \mathcal{S}_{p}\left(R_{p}\right)$ and let $y(t)=\mathcal{Q}_{F, H}(x(t))$. From the remarks concerning $\mathcal{Q}_{F, H}$ done after its definition, at the end of the previous section, $y(t+p)=$ $\mathcal{Q}_{F, H}(x(t+p))=\mathcal{Q}_{F, H}(x(t))=y(t)$ and there exists no $d<p$ such that $y(t+d)=y(t)$ (otherwise, from the injectivity of $\mathcal{Q}_{F, H}$ it would hold that $x(t+d)=x(t)$ which contradicts $\left.x(t) \in \mathcal{S}_{p}\left(R_{p}\right)\right)$.

Thus, provided $R_{p}=\left(\mathbb{C}_{p}, F\right)$ is positive, $\mathrm{A}_{p}\left(R_{p}\right)$ is independent of the distribution and number of negative arcs in $\mathbb{C}_{p}$. Therefore, from now on, we will use the following notations:

$$
\mathrm{A}_{p}^{+}=\frac{1}{p} \cdot\left|\mathcal{S}_{p}\left(R_{p}\right)\right|=\mathrm{A}_{p}\left(R_{p}\right)
$$

for all positive Boolean automata circuits $R_{p}$ of size $p$.

Lemma 4. Let $R_{n}$ be a positive Boolean automata circuit of size $n$. Then, for every divisor $p$ of $n$,

$$
A_{p}\left(R_{n}\right)=A_{p}^{+}
$$


Proof. Suppose that $R_{n}=\left(\mathbb{C}_{n}, F\right)$ where $F$ is defined by $\left\{f_{i} \mid i \in \mathbb{Z} / n \mathbb{Z}\right\}$ and $n=q \cdot p, q, p \in \mathbb{N}$. We will show that there exists a network $R_{p}$ of size $p$ such that the sets $\mathcal{S}_{p}\left(R_{n}\right)$ and $\mathcal{S}_{p}\left(R_{p}\right)$ are isomorphic. First, we define the network $R_{p}=\left(\mathbb{C}_{p}, H\right)$ where $H$, the global transition function of $R_{p}$, is defined by $\left\{h_{0}=F[0, p]\right\} \cup\left\{h_{i}=f_{i} \mid 0<i<p\right\}$.

Now, suppose that $x(t)$ belongs to $\mathcal{S}_{p}\left(R_{p}\right)$ and let $y(t)=\mathcal{Q}_{F, H}(x(t)) \in$ $\{0,1\}^{n}$. By an argument similar to that used in the proof of Lemma 3, we find that $y(t) \in \mathcal{S}_{p}\left(R_{n}\right)$.

On the other hand, if $y(t) \in \mathcal{S}_{p}\left(R_{n}\right)$, we define $x(t) \in\{0,1\}^{p}$ such that $\forall i \in$ $\mathbb{Z} / p \mathbb{Z}, x_{i}(t)=y_{i}(t)$. Because $\mathbb{C}_{n}$ and $\mathbb{C}_{p}$ are positive and because $h_{0}=F[0, p]$, $\mathcal{P}_{F}\left(p, y(t)\right.$ ) (true by Lemma 1) implies $y(t)=\mathcal{Q}_{F, H}(x(t)$ ). Lemma 2 suffices to state that $x(t+p)=x(t)$. But it is, again, by the injectivity of $\mathcal{Q}_{F, H}$ that we can claim that there is no $d<p$ such that $x(t+d)=x(t)$.

As a consequence of the previous lemmas, we finally get the main result of this section:

Theorem 1. $\forall n \in \mathbb{N}$,

(i) $2^{n}=\sum_{p \mid n} A_{p}^{+} \times p$

(ii) $A_{n}^{+}=\frac{1}{n} \cdot \sum_{p \mid n} \mu\left(\frac{n}{p}\right) \cdot 2^{p}$

(iii) $T_{n}^{+}=\frac{1}{n} \cdot \sum_{p \mid n} \psi\left(\frac{n}{p}\right) \cdot 2^{p}$

where $\mu$ is the Möbius function [25,26], $\psi$ the Euler totient function and $T_{n}^{+}$the total number of distinct attractors of a positive Boolean automata circuit.

Proof. Let $R_{n}$ be a positive Boolean automata circuit. By Lemma 2, all of the $2^{n}$ global states of a $R_{n}$ belong to an attractor whose period is a divisor of $n$. (i) then comes from Lemmas 3 and 4 . (ii) is shown using the Möbius inversion formula (see $[25,26])$ on (i). For the proof of (iii), we use the fact that $\psi(m)=$ $\sum_{r \mid m}(m / r) \cdot \mu(r)$ :

$$
\begin{aligned}
& \mathrm{T}_{n}^{+}= \sum_{p \mid n} \mathrm{~A}_{p}^{+}=\sum_{p \mid n} \sum_{d \mid p} \frac{1}{p} \cdot \mu\left(\frac{p}{d}\right) \cdot 2^{d} \\
&=\frac{1}{n} \cdot \sum_{p \mid n} \sum_{d \mid p} 2^{d} \cdot \frac{n}{p} \cdot \mu\left(\frac{p}{d}\right)=\frac{1}{n} \cdot \sum_{p \mid n} \sum_{d \mid p} 2^{d} \cdot \frac{n}{(p / d) \cdot d} \cdot \mu\left(\frac{p}{d}\right) \\
& \quad=\frac{1}{n} \cdot \sum_{d \mid n} 2^{d} \sum_{k \mid n / d} \frac{n}{k \cdot d} \cdot \mu(k)=\frac{1}{n} \cdot \sum_{d \mid n} \psi\left(\frac{n}{d}\right) \cdot 2^{d} .
\end{aligned}
$$

In particular, point (ii) of Theorem 1 implies that if $n$ is prime then, since $\mu(n)=-1$,

$$
\mathrm{A}_{n}^{+}=\frac{1}{n} \cdot\left(\mu(n) \cdot 2+\mu(1) \cdot 2^{n}\right)=\frac{2^{n}-2}{n} .
$$


Notice also that because 1 is a divisor of all $n \in \mathbb{N}$, every positive Boolean automata circuit $R_{n}$ has exactly two fixed points, i.e, $\mathrm{A}_{1}\left(R_{n}\right)=\mathrm{A}_{1}\left(R_{1}\right)=\mathrm{A}_{1}^{+}=2$. In [5] and [27], positive circuits are characterised this way and indeed, as we will see in the next section, negative circuits never have any fixed points. From this characterisation, the authors of these articles also infer some results concerning more general networks.

Table 1. Number of attractors of positive Boolean automata circuits.

\begin{tabular}{|c|c|c|c|c|c|c|c|c|c|c|c|c|c|c|}
\hline$p{ }^{n}$ & 1 & 2 & 3 & 4 & 5 & 6 & 7 & 8 & 9 & 10 & 11 & 12 & 21 & 22 \\
\hline 1 & 2 & 2 & 2 & 2 & 2 & 2 & 2 & 2 & 2 & 2 & 2 & 2 & 2 & 2 \\
\hline 2 & - & 1 & - & 1 & - & 1 & - & 1 & - & 1 & - & 1 & - & 1 \\
\hline 3 & - & - & 2 & - & - & 2 & - & - & 2 & - & - & 2 & 2 & - \\
\hline 4 & - & - & - & 3 & - & - & - & 3 & - & - & - & 3 & - & - \\
\hline 5 & - & - & - & - & 6 & - & - & - & - & 6 & - & - & - & - \\
\hline 6 & - & - & - & - & - & 9 & - & - & - & - & - & 9 & - & - \\
\hline 7 & - & - & - & - & - & - & 18 & - & - & - & - & - & 18 & - \\
\hline 8 & - & - & - & - & - & - & - & 30 & - & - & - & - & - & - \\
\hline 9 & - & - & - & - & - & - & - & - & 56 & - & - & - & - & - \\
\hline 10 & - & - & - & - & - & - & - & - & - & 99 & - & - & - & - \\
\hline 11 & - & - & - & - & - & - & - & - & - & - & 186 & - & - & 186 \\
\hline 12 & - & - & - & - & - & - & - & - & - & - & - & 335 & - & - \\
\hline & & & & & & & & & & & & & & \\
\hline 21 & - & - & - & - & - & - & - & - & - & - & - & - & 99858 & - \\
\hline 22 & - & - & - & - & - & - & - & - & - & - & - & - & - & 190557 \\
\hline $\mathrm{T}_{n}^{+}$ & 2 & 3 & 4 & 6 & 8 & 14 & 20 & 36 & 60 & 108 & 188 & 352 & 99880 & 190746 \\
\hline
\end{tabular}

We performed computer simulations of the dynamical behaviour of positive circuits of sizes between 1 and 22. Simulations done for different circuits of the same size confirmed Lemma 3. An example picturing these results is given in Figure 1 where two different positive circuits of size 4 and their dynamics are represented. Table 1 shows some of the results we obtained for circuits of different sizes. In this table, $n$ is the size of the network and $p$ the period of the attractor. In the cell corresponding to line $p$ and column $n$ figures $\mathrm{A}_{p}\left(R_{n}\right)$. Notice that as Lemma 4 predicts, all numbers appearing on one line are the same. In particular, line one indicates that all positive circuits have two fixed points.

\section{Negative circuits}

We are now going to consider negative circuits. The approach we take here is very similar to that of the previous section so we will give few comments on how this case is handled. We may note incidently that most results concerning negative Boolean automata circuits can be derived from those concerning positive Boolean automata circuits by associating to a negative Boolean automata circuit $R_{n}=\left(\mathbb{C}_{n}, F\right)$, the positive Boolean automata circuit $R_{2 n}=\left(\mathbb{C}_{2 n}, H\right)$ where $H$ is defined by the set $\left\{h_{i} \mid i \in \mathbb{Z} / 2 n \mathbb{Z}, h_{i}=h_{i+n}=f_{i}\right\}$. Just as Lemma 2 does for the positive case, Lemma 5 recalls and extends some important general 
properties of the dynamics of negative circuits that were mentioned by Goles et al. in [24].

Lemma 5. Let $R_{n}=\left(\mathbb{C}_{n}, F\right)$ be a negative Boolean automata circuit of size $n$. Then,

1. Every global state $x \in\{0,1\}^{n}$ of $R_{n}$ belongs to an attractor of period a divisor of $2 n$;

2. If $\mathcal{S}_{p}\left(R_{n}\right) \neq \emptyset$, then $p$ is an even divisor of $2 n$ and $n=q \times \frac{p}{2}$ where $q$ is odd.

Proof.

1. By a similar proof to that of Lemma 2 , we find that $\forall x(t) \in\{0,1\}^{n}$, $x(n+t)=\neg x(t)$ which implies that $x(2 n+t)=x(t)$. Thus every global state belongs to an attractor of period a divisor of $2 n$.

2. Suppose that $x \in \mathcal{S}_{p}\left(R_{n}\right)$ where $p$ divides $n$. By Lemma $1, \mathcal{P}_{F}(p, x)$ must be true so $\forall 0 \leq r<p, x_{r}=F[r, r-p+1]\left(x_{r-p}\right)=F[r, r-p+1] \circ F[r-p, r+$ $1]\left(x_{r}\right)=F[r, r+1]\left(x_{r}\right)$. However, because $\mathbb{C}_{n}$ is negative, $F[r, r+1]=$ neg. This leads to the contradiction $x_{r}=\neg x_{r}$. Thus, if $\mathcal{S}_{p}\left(R_{n}\right) \neq \emptyset$, then $p$ divides $2 n$ without dividing $n$. This means that $p$ must be even and we necessarily must have $n=q \times p / 2$ where $q$ is odd.

Analog results of Lemmas 3 and 4 for the negative case can be shown. They allow us to note, for all negative Boolean automata circuit $R_{n}$ where $n=p \times q$ and $q$ is odd,

$$
\mathrm{A}_{2 p}^{-}=\frac{1}{2 p} \cdot\left|\mathcal{S}_{2 p}\left(R_{n}\right)\right|=\mathrm{A}_{2 p}\left(R_{n}\right) .
$$

As a consequence, we obtain the following theorem which is proven with very similar arguments to that used in the proof of Theorem 1.

Table 2. Number of attractors of negative Boolean automata circuits.

\begin{tabular}{|c|c|c|c|c|c|c|c|c|c|c|c|c|c|c|}
\hline$p 5^{n}$ & 1 & 2 & 3 & 4 & 5 & 6 & 7 & 8 & 15 & 16 & 17 & 18 & 21 & 22 \\
\hline 2 & 1 & - & 1 & - & 1 & - & 1 & - & 1 & - & 1 & - & 1 & - \\
\hline 4 & - & 1 & - & - & - & 1 & - & - & - & - & - & 1 & - & 1 \\
\hline 6 & - & - & 1 & - & - & - & - & - & 1 & - & - & - & 1 & - \\
\hline 8 & - & - & - & 2 & - & - & - & - & - & - & - & - & - & - \\
\hline 10 & - & - & - & - & 3 & - & - & - & 3 & - & - & - & - & - \\
\hline 12 & - & - & - & - & - & 5 & - & - & - & - & - & 5 & - & - \\
\hline 14 & - & - & - & - & - & - & 9 & - & - & - & - & - & 9 & - \\
\hline 16 & - & - & - & - & - & - & - & 16 & - & - & - & - & - & - \\
\hline & & & & & & & & & & & & & & \\
\hline 30 & - & - & - & - & - & - & - & - & 1091 & - & - & - & - & - \\
\hline 32 & - & - & - & - & - & - & - & - & - & 2048 & - & - & - & - \\
\hline 34 & - & - & - & - & - & - & - & - & - & - & 3855 & - & - & - \\
\hline 36 & - & - & - & - & - & - & - & - & - & - & - & 7280 & - & - \\
\hline & & & & & & & & & & & & & & \\
\hline 42 & - & - & - & - & - & - & - & - & - & - & - & - & 49929 & - \\
\hline 44 & - & - & - & - & - & - & - & - & - & - & - & - & - & 95325 \\
\hline $\mathrm{T}_{n}^{-}$ & 1 & 1 & 2 & 2 & 4 & 6 & 10 & 16 & 1096 & 2048 & 3856 & 7286 & 49940 & 95326 \\
\hline
\end{tabular}


Theorem 2. $\forall n \in \mathbb{N}$,

(i) $2^{n}=\sum_{\text {odd } q \mid n} A_{2 n / q}^{-} \times 2 n / q$

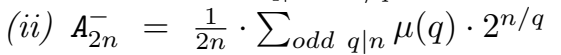

(iii) $T_{n}^{-}=\frac{1}{2 n} \cdot \sum_{o d d p \mid n} \psi\left(\frac{n}{p}\right) \cdot 2^{p}$

where $T_{n}^{-}$is the total number of distinct attractors of a negative Boolean automata circuit (and where $\mu$ and $\psi$ still are, respectively, the Möbius function and the Euler totient as in theorem 1).

Computer simulations of the dynamics of negative circuits of sizes between 1 and 22 were performed. The results of these simulations are shown in Table 2 below (see the last paragraph of section 3 for an explanation of what holds each cell of this table). A particular case of formulas (ii) and (iii) of Theorem 2 is when $n=2^{k}$. Then, since 1 is the only odd divisor of $n, \mathrm{~A}_{2 n}^{-}=\mathrm{T}_{n}^{-}=2^{n-k-1}$ (see cells $(p=16, n=8)$ and $(p=32, n=8)$ of Table 2$)$.

Again, we may also note that Theorem 2 implies that a negative circuit never has any fixed points in its dynamics. In [5] and [27], the authors proven a result stating that arbitrary networks containing only negative circuits have no fixed points.

\section{Related problems}

Thanks to the results of the previous sections, it is possible to define and concentrate on one canonical circuit of each sign and size. Indeed, for the positive case, for instance, since $\mathrm{A}_{p}^{+}$does not depend on the distribution or number of negative arcs in the circuit in question as long as this number is even, it is possible to choose a positive circuit $R_{n}^{+}=\left(\mathbb{C}_{n}, F^{+}\right)$as the representative of all positive circuits of same size $n$. Then, for any other positive network $R_{n}=\left(\mathbb{C}_{n}, F\right)$, there exists a permutation $\sigma$ of $\{0,1\}^{n}$ such that $\forall x \in\{0,1\}^{n}, F(x)=F^{+}(\sigma(x))$. Choosing a canonical positive circuit is easy and straightforward: $R_{n}^{+}=\left(\mathbb{C}_{n}, F^{+}\right)$ can be the circuit of size $n$ that has no negative arcs. Note that $F^{+}$is then defined by a set of $n$ local functions all equal to $i d$ and acts as a rotation of the coefficients of vectors in $\{0,1\}^{n}$ :

$$
\forall\left(x_{0}, \ldots, x_{n-1}\right) \in\{0,1\}^{n}, F^{+}\left(x_{0}, \ldots, x_{n-1}\right)=\left(x_{n-1}, x_{0}, \ldots, x_{n-2}\right) .
$$

Choosing wisely a canonical negative circuit is less obvious because a circuit with only negative arcs is negative only if its size is odd. The choice of this circuit must therefore depend on the use we want to make of it.

Now, sequences $\left(\mathrm{A}_{n}^{+}\right)_{n \in \mathbb{N}},\left(\mathrm{T}_{n}^{+}\right)_{n \in \mathbb{N}},\left(\mathrm{A}_{2 n}^{-}\right)_{n \in \mathbb{N}}$ and $\left(\mathrm{T}_{n}^{-}\right)_{n \in \mathbb{N}}$ defined in the previous sections happen to correspond precisely and respectively to the integer sequences A1037, A31, A48 and A16 of the OEIS [28]. In [28], these sequences are defined by different combinatoric problems. Defining canonical Boolean automata circuits allowed us to study how some of them relate to the problem of counting the number of attractors (of given period or in total) of Boolean 
automata circuits. In particular we focused on those problems related to binary necklaces and Lyndon words [29], to binary shift register sequences [30,31] and to cycles in a digraph under $x^{2} \bmod q$ where $q=2^{n+1}-1$ is a Mersenne prime [32]. The relationships found with these problems, provided interesting different ways of formalising the dynamics of Boolean automata circuits updated synchronously. For instance, the work presented in $[30,31]$ allowed us to formalise the dynamics of circuits in terms of the action of a permutation group on the set of global states and corroborate the formulas for $\mathrm{T}_{n}^{+}$and $\mathrm{T}_{n}^{-}$using the Burnside Lemma [33,34]. Drawing inspiration from [32], we also derived a new expression for $\mathcal{S}_{p}\left(R_{n}^{+}\right)$:

$$
\mathcal{S}_{p}\left(R_{n}^{+}\right)=\left\{x \in G \mid \operatorname{ord}(x)=d \text { and } p=\operatorname{ord}_{d}(2)\right\}
$$

where $d=\operatorname{ord}(x)=\min \left\{k \mid x \cdot k \equiv 0 \bmod 2^{n}-1\right\}$ is the order of $x$ in the cyclic additive group $\mathbb{Z} /\left(2^{n}-1\right) \mathbb{Z}$ and $\operatorname{ord}_{d}(2)$ is the order of 2 in the group $(\mathbb{Z} / d \mathbb{Z})^{*}$. And since $\psi(d)$ counts the number of elements of order $d$ in $\mathbb{Z} /\left(2^{n}-1\right) \mathbb{Z}$, we thus obtain another way of explaining the presence of the Euler totient function in the formula of $\mathrm{T}_{n}^{+}$.

\section{Conclusion}

From the results presented in this article, several questions arise. The first and most obvious of them is, now that we know the dynamics of circuits synchronously updated, how do these results translate into other update schedules such as sequential or block-sequential schedules for instance?

Besides its obvious need for an extension towards more general update schedules, we believe our work would also benefit from an extension toward more general networks such as feedforward networks and perhaps even random networks. Further, comparisons with other related studies and the results they produced are called for. For instance, in [2], [35] and in [5], experimental or theoretical results prove or suggest that the networks in question have only very little different asymptotic dynamical behaviours $(\mathcal{O}(\sqrt{n})$ in the case of connectivity 2 networks considered in [2] and [5], one or two in the case of the small networks studied in [35]). This seems, at first sight, to be in contradiction with the exponential number of attractors of Boolean automata circuits that we found above. It would be interesting to connect the two sources of results in order to lift the contradiction. Thus, relating the dynamics of a network with that of the circuits it contains when they are isolated seems to be a natural and essential step in the comprehension of the dynamics of ordinary Boolean automata networks.

\section{Acknowledgement}

Many thanks to Éric Rémila for his useful comments. 


\section{References}

1. McCulloch, W.S., Pitts, W.: A logical calculus of the ideas immanent in nervous activity. Journal of Mathematical Biology 5 (1943) 115-133

2. Kauffman, S.A.: Metabolic stability and epigenesis in randomly constructed genetic nets. Journal of Theoretical Biology 22 (1969) 437-467

3. Thomas, R.: Boolean formalisation of genetic control circuits. Journal of Theoretical Biology 42 (1973) 563-585

4. Snoussi, E.H.: Necessary conditions for multistationarity and stable periodicity. Journal of Biological Systems 6 (1998) 3-9

5. Aracena, J., Ben Lamine, S., Mermet, O., Cohen, O., Demongeot, J.: Mathematical modeling in genetic networks: relationships between the genetic expression and both chromosomic breakage and positive circuits. IEEE Transactions on Systems, Man, and Cybernetics 33 (2003) 825-834

6. Soulé, C.: Mathematical approaches to differentiation and gene regulation. Comptes rendus de l'Académie des sciences, Biologies 329 (2006) 13-20

7. Kaufman, M., Soulé, C., Thomas, R.: A new necessary condition on interaction graphs for multistationarity. Journal of Theoretical Biology 248 (2007) 675-685

8. Rémy, E., Ruet, P., Thieffry, D.: Graphic requirements for multistability and attractive cycles in a boolean dynamical framework. Advances in Applied Mathematics 41 (2008) 335-350

9. Hopfield, J.J.: Neural networks and physical systems with emergent collective computational abilities. Proceedings of the National Academy of Sciences of the USA 79 (1982) 2554-2558

10. Hopfield, J.: Neurons with graded response have collective computational properties like those of two-state neurons. Proceedings of the National Academy of Sciences of the USA 81 (1984) 3088-3092

11. Goles, E., Olivos, J.: Comportement périodique des fonctions à seuils binaires et applications. Discrete Applied Mathematics 3 (1981) 93-105

12. Goles, E.: Fixed point behavior of threshold functions on a finite set. SIAM Journal on Algebraic and Discrete Methods 3 (1982) 529-531

13. Fogelman-Soulie, F., Goles, E., Weisbuch, G.: Transient length in sequential iteration of threshold functions. Discrete Applied Mathematics 6 (1983) 95-98

14. Thomas, R.: On the relation between the logical structure of systems and their ability to generate multiple steady states or sustained oscillations. Springer Series in Synergetics 9 (1981) 180-193

15. Kauffman, S.A.: The origins of order: self-organization and selection in evolution. Oxford University Press (1993)

16. Hilbert, D.: Mathematical problems. Bulletin of the American Mathematical Society 8 (1902) 437-479

17. Aracena, J., Demongeot, J., Goles, E.: On limit cycles of monotone functions with symmetric connection graph. Theoretical Computer Science 322 (2004) 237-244

18. Aracena, J., Demongeot, J., Goles, E.: Fixed points and maximal independent sets in and-or networks. Discrete Applied Mathematics 138 (2004) 277-288

19. Richard, A., Comet, J.: Necessary conditions for multistationarity in discrete dynamical systems. Discrete Applied Mathematics 155(18) (2007) 2403-2413

20. Demongeot, J., Jézéquel, C., Sené, S.: Boundary conditions and phase transitions in neural networks. theoretical results. Neural Networks 21(7) (2008) 971-979

21. Cangiano, L.: Mechanisms of rhythm generation in the lamprey locomotor network. PhD thesis, Karolinska Institute, Stockholm (2004) 
22. Grillner, S.: The motor infrastructure: from ion channels to neuronal networks. Nature Reviews Neuroscience 4 (2003) 573-586

23. Müller, T.H., Swandulla, D., Zeilhofer, H.U.: Synaptic connectivity in cultured hypothalamic neuronal networks. Journal of Neurophysiology 77(6) (1997) 32183225

24. Goles, E., Fogelman-Soulie, F., Weisbuch, G.: Specific roles of different boolean mappings in random networks. Bulletin of Mathematical Biology 44(5) (1982) $715-730$

25. Gauss, C.F., Clarke, A.A.t.: Disquisitiones Arithemeticae. Yale University Press (1965)

26. Apostol, T.M.: Introduction to analytic number theory. Springer-Verlag (1976)

27. Aracena, J.: Discrete mathematics models associated to biological systems. Application to the genetic regulatory networks. PhD thesis, Université Joseph Fourier Grenoble (2001)

28. Sloane, N.J.A.: The On-Line Encyclopedia of Integer Sequences (2008)

29. Ruskey, F.: Information on necklaces, unlabelled necklaces, Lyndon words, De Bruijn sequences

30. Golomb, S.W.: Shift register sequences. Aegean Park Press, Laguna Hills, CA, USA (1981)

31. Sloane, N.J.A.: On single-deletion-correcting codes. In Arasu, K.T., Seress, A., eds.: Codes and Designs, de Gruyter, Berlin (2002) 273-291

32. Vasiga, T., Shallit, J.: On the iteration of certain quadratic maps over GF(p). Discrete Mathematics 277 (2004) 219-240

33. Burnside, W.: Theory of groups of finite order. Cambridge University Press (1897)

34. Frobenius, F.G.: Ueber die Congruenz nach einem aus zwei endlichen Gruppen gebildeten Doppelmodul. Crelle CI: 288 (1887)

35. Elena, A.: Robustesse des réseaux d'automates booléens à seuil aux modes d'itération. Application à la modélisation des réseaux de régulation génétique. $\mathrm{PhD}$ thesis, Université Joseph Fourier - Grenoble (2009) 